\title{
Risk factors among young women with endometrial cancer: A Danish case-control study
}

\author{
Michael Parslov, MD, a Øjvind Lidegaard, MD, DSci, a Søren Klintorp, MD, b Beth Pedersen, MD,b \\ Lisbeth Jønsson, MD,c Poul S. Eriksen, MD, DSci,c and Bent 0 ttesen, MD, DScib \\ Copenhagen, Denmark
}

OBJECTIVE: This study was undertaken to identify and quantify risk factors for endometrial cancer among young women.

STUDY DESIGN: This case-control study included all Danish women $<50$ years old who had endometrial cancer diagnosed during the period 1987 to 1994 . A total of 237 case patients and 538 population control subjects matched with the case patients for age and residence were included in the analysis.

RESULTS: Women with a family history of endometrial cancer had an odds ratio for endometrial cancer of 2.1 (95\% confidence interval, 1.1-3.8)). Completion of 1 term pregnancy implied an odds ratio of 0.6 (95\% confidence interval, $0.3-1.1)$. The risk of endometrial cancer decreased significantly with increasing age at first birth and with the number of induced abortions. Use of oral contraceptives for 1 to 5 years decreased the risk of endometrial cancer (odds ratio, $0.2 ; 95 \%$ confidence interval, $0.1-0.3$ ). The odds ratio for endometrial cancer among women who received hormone replacement therapy for 1 to 5 years was 3.1 ( $95 \%$ confidence interval, 1.4-7.0). Body mass index was not demonstrated to be an independent risk factor in this study. The protective impacts of the different exposures (risk factors) can be expressed as etiologic fractions, which indicate how much each exposure reduces the occurrence of endometrial cancer compared with a situation without the existence of that particular exposure. These fractions were as follows: oral contraceptive use for $\geq 1$ year, $-45 \%$; 2 term pregnancies, $-88 \%$; age $\geq 30$ y when giving birth for the first time, $-38 \%$; and a history of incomplete pregnancy, $-16 \%$.

CONCLUSION: A number of risk factors for endometrial cancer are common to premenopausal and postmenopausal women: family history, reproductive history, hormone replacement therapy, and the use of oral contraceptives. Among young women reproductive variables imply the greatest prophylactic potential. (Am J Obstet Gynecol 2000;182:23-9.)

Key words: Case-control study, endometrial cancer, etiologic fraction, premenopausal, risk factor

Endometrial cancer is a rare disease among premenopausal women. Seven percent of endometrial cancer occurs in women $<50$ years old. In the same age group about 300 curettage procedures and an equal number of endometrial biopsies are performed for every single diagnosis of endometrial cancer. When the physical complications attendant with these invasive procedures and the socioeconomic consequences of curettage as a screening procedure are considered, the question

From the Department of O bstetrics and Gynecology, $\mathrm{H}$ erlev $\mathrm{H}$ ospital, a the Department of Obstetrics and Gynecology, H vidovre Hospital,b University of Copenhagen, and the Department of Obstetrics and Gynecology, Naestved H ospital.c

Supported by grants from The Medical Research Foundation in Frederiksborg County, The Danish Foundation for Advancement of $M$ edical Science, NovoCare Foundation, and M anager Jacob M adsen and wife Olga M adsen's Foundation.

Received for publication September 18, 1998; revised June 25, 1999; accepted August 31, 1999.

Reprints not available from the authors.

Copyright $\odot 2000$ by M osby, Inc.

$0002-9378 / 2000 \$ 12.00+0 \quad 6 / 1 / 102706$ arises as to whether it is appropriate to carry out this number of intrauterine procedures in fertile women to find so few cases of endometrial malignancy.

If young women at an increased risk for development of endometrial cancer could be identified, a selection of women among whom the need to undergo curettage was greater might be realized. Consequently, the number of curettage procedures performed in this age group could be reduced.

Several studies have identified risk factors of endometrial cancer among older women, such as overweight status, estrogen replacement therapy, nulliparity, infertility, diabetes mellitus, and hypertension. ${ }^{1-8} \mathrm{H}$ owever, these risk factors are not necessarily relevant for en dometrial cancer among young women. O nly a few studies have focused on risk factorsfor endometrial cancer among premenopausal women (Table I). To identify and quantify risk factors for endometrial cancer among premenopausal women, we conducted a case-control study that included all Danish women aged 25 to 49 years who had endometrial cancer diagnosed during an 8-year period. 
Table I. Studies on risk factors of endometrial cancer among young women

\begin{tabular}{|c|c|c|c|c|}
\hline Study & $N$ ationality & Sampling period & Age (y) & $\begin{array}{l}\text { Ratio of case patients } \\
\text { to control subjects }\end{array}$ \\
\hline Henderson et al9 & United States & 1972-1979 & $\leq 45$ & $127 / 127$ \\
\hline La Vecchia et al8* & Italy & $1979-1983$ & $\leq 50$ & $30 / 94$ \\
\hline Smith et al $10 *$ & United States & $1980-1982$ & $\leq 49$ & $39 / 419$ \\
\hline Tyler et al11* & United States & $1980-1982$ & $\leq 49$ & $206 / 2355$ \\
\hline World H ealth Organization $12 *$ & Multinational & $1979-1986$ & $25-59$ & $135 / 835$ \\
\hline Dahlgren et al 13 & Sweden & $1968-1978$ & $31-45$ & $68 / 761$ \\
\hline Parazzini et al7* & Italy & $1983-1988$ & $\leq 49$ & $66 / 769$ \\
\hline Parslov et al (current study) & Denmark & 1987-1994 & $25-49$ & $237 / 538$ \\
\hline
\end{tabular}

*Study included all age groups, but data for young women could be separated from those of older women, thus allowing interpretation of results specifically for young women. The number of case patients includes only case patients within the age range indicated.

Table II. Inclusion and exclusion of case patients with endometrial cancer and control subjects

\begin{tabular}{lcc}
\hline & Case patients & Control subjects \\
\hline Identified subjects & 384 & 642 \\
Excluded & & - \\
$\quad$ Death after discharge from department & 11 & - \\
$\quad$ Wrong diagnosis (message from hospital) & 52 & - \\
Permission to contact patient not granted & 2 & $642(100 \%)$ \\
Submitted questionnaires & $319(100 \%)$ & $586(91.3 \%)$ \\
Returned questionnaires & $295(92.5 \%)$ & - \\
Excluded questionnaires & 6 & - \\
$\quad$ Wrong diagnosis (message from case patient) & 29 & 16 \\
$\quad$ Wrong diagnosis (according to histologic report) & 23 & 3 \\
$\quad$ Refusal to participate & - & 538 \\
$\quad$ Hysterectomy of potential control subject & - & 237 \\
Other & & \\
Included questionnaires & & \\
\hline
\end{tabular}

\section{Material and methods}

Case patients. All Danish women 25 to 49 years old who had endometrial cancer during the period 1987 to 1994 (International Classification of Diseases, Eighth Revision diagnosis 182.00-182.09 [1987-1993], International Classification of Diseases, Tenth Revision diagnosis C54.0C54.9 [1994]) were identified in the Danish National Patient Register. Restriction for readmissions was ensured. Women with previous malignant genital disease were identified in the register and primarily excluded. The specific diagnosis was confirmed by review of the histologic reports in the hospital records.

Written permission was obtained from all 45 hospital departments involved to send a questionnaire to the affected women. The questionnaire included information about family history of endometrial cancer (mother or sister), body weight, height, diabetes mellitus (medically treated), hypertension (medically treated), menarche, parity, induced or spontaneous abortions, extrauterine pregnancies, infertility, hyperandrogenism, amenorrhea (>3 months, excluding pregnancy and menopause), length of use of oral contraceptives, length of use of intrauterine contraceptive device, length of use of hormone replacement therapy, previous curettage, cigarette smoking, years of schooling, and other previous cancer- ous diseases. Nonresponders received a written reminder twice with 3-week interval.

A total of 384 case patients were identified. Sixty-five were excluded because of either death since discharge ( $n$ $=11$ ), wrong diagnosis according to the department ( $n=$ 52 ), or missing permission to contact the patient $(n=2)$. Questionnaires were sent out to 319 women, and 295 $(92.5 \%)$ of these were returned. Among the 295 responders 23 refused to participate, 6 denied having had endometrial cancer, and in 29 cases the histologic diagnosis could not confirm the diagnosis in the register. The remaining 237 case patients were included in the analysis (Table II).

Control subjects. For each case patient identified during the period of 1987 through 1992, 3 female control subjects were randomly selected from the Central Person Register, which includes all Danish inhabitants. The control subjects were matched by the age of the case patient at the time of diagnosis and by the geographic region. The control questionnaires included the same information as for case patients. In addition, the control subjects were asked whether they ever had endometrial cancer; those who replied in the affirmative were excluded.

In total, 642 control questionnaires were sent out, of 
Table III. Distribution of case patients and control subjects according to investigated risk factors

\begin{tabular}{|c|c|c|c|c|}
\hline \multirow[b]{2}{*}{ Characteristic } & \multicolumn{2}{|c|}{ Case patients } & \multicolumn{2}{|c|}{ Control subjects } \\
\hline & No. & $\%$ & No. & $\%$ \\
\hline Age ( $y$, mean and range) & $44(27-49)$ & & $43(25-49)$ & \\
\hline Age $25-29$ y ( No.) & 3 & 1.3 & 18 & 3.3 \\
\hline Age $30-34$ y (No.) & 5 & 2.1 & 21 & 3.9 \\
\hline Age $35-39$ y ( No.) & 22 & 9.3 & 83 & 15.5 \\
\hline Age $40-44$ y ( No.) & 63 & 26.6 & 177 & 32.9 \\
\hline Age $45-49$ y (No.) & 144 & 60.7 & 239 & 44.4 \\
\hline Body mass index $\left(\mathrm{kg} / \mathrm{m}^{2}\right.$, mean and range) & $25.3(14-66)$ & & $23.5(16-43)$ & \\
\hline Body mass index $<20 \mathrm{~kg} / \mathrm{m}^{2}$ (No.) & 19 & 8.0 & 50 & 9.3 \\
\hline Body mass index $20-24$ kg/ m²(No.) & 131 & 55.3 & 328 & 61.0 \\
\hline Body mass index $25-29 \mathrm{~kg} / \mathrm{m}^{2}$ ( No.) & 45 & 19.0 & 118 & 22.0 \\
\hline Body mass index $\geq 30 \mathrm{~kg} / \mathrm{m}^{2}$ ( $\mathrm{No}$.) & 42 & 17.7 & 42 & 7.7 \\
\hline H ormone replacement therapy (ever used) & 39 & 16.5 & 50 & 9.3 \\
\hline Oral contraceptives (ever used) & 147 & 62.0 & 463 & 86.1 \\
\hline Menarche ( $y$, mean and range) & $13.2(9-18)$ & & $13.4(9-17)$ & \\
\hline Amenorrhea (ever) & 39 & 16.5 & 55 & 10.2 \\
\hline Pregnancy (ever) & 192 & 81.0 & 500 & 92.9 \\
\hline Pregnancies (mean and range) & $2.0(0-6)$ & & $2.5(0-7)$ & \\
\hline Births (mean and range) & $1.5(0-5)$ & & $1.8(0-5)$ & \\
\hline Infertility (ever) & 42 & 17.7 & 80 & 14.9 \\
\hline Diabetes mellitus & 4 & 1.7 & 3 & 0.6 \\
\hline H ypertension & 16 & 6.8 & 19 & 3.5 \\
\hline Cigarette smoking (ever) & 134 & 56.5 & 348 & 64.7 \\
\hline Family history of endometrial cancer & 23 & 9.7 & 31 & 5.8 \\
\hline Induced abortions (mean and range) & $0.2(0-2)$ & & $1.8(0-5)$ & \\
\hline Extrauterine pregnancies (mean and range) & $0.02(0-1)$ & & $0.04(0-2)$ & \\
\hline Hyperandrogenism & 7 & 3.0 & 3 & 0.6 \\
\hline Intrauterine contraceptive device use (ever) & 84 & 35.4 & 258 & 48 \\
\hline
\end{tabular}

which 586 (91.3\%) were returned. Exclusions were made for mental disease, ${ }^{1}$ H untington chorea, ${ }^{1}$ and congenital agenesis of internal genitalia1; another 16 refused to participate, and 29 reported a history of hysterectomy. Except for 1 patient with cervical cancer, all control subjects who had undergone hysterectomy stated that the operation had been done for a benign indication. The remaining 538 control subjects were included in the analysis (Table II).

Statistics. The conditional distribution of risk factors and potential confounders that would be effect modifiers, given case-control status and age, was analyzed by log-linear graphic models for multidimensional contingencies. ${ }^{14}$ Statistical tests included likelihood ratio tests, $\chi^{2}$ tests, and partial $\gamma$ coefficients measuring the association between either ordinal variables or binary variables or both ordinal and binary variables.

Risk estimates were calculated as odds ratios with $95 \%$ confidence intervals with adjustment for influence of investigated confounders. Such odds ratios are good estimates of the relative risk associated with each risk factor because the investigated disease is rare. Combining the relative risk with the prevalence of the specific exposition allows calculation of the etiologic fraction for each risk factor. The etiologic fraction indicates the proportion of endometrial cancer that would disappear if the risk factor were eliminated.

\section{Results}

Characteristics of the study population are presented in Table III. The mean age of case patients was 44 years, with 30 patients ( $12.7 \%$ ) $<40$ years old. The mean age of control subjects was 43 years. The difference between numbers of case patients and control subjects in the different age groups was because of exclusions of case patients and control subjects. In addition, the control subjects were matched with case patients from the 6-year period 1987 through 1992, whereas case patients also included women with disease diagnosed in 1993 and 1994. In the multivariate analysis, adjustment was therefore made for these small age differences.

A marginal (crude) difference between case patients and control subjects was observed for all variables except menarche. However, controlling for confounding influence from the other determinants revealed the following variables to be independent risk factors: family history of endometrial cancer, parity, age at first birth, number of induced abortions, use of oral contraceptives, and use of hormone replacement therapy (Table IV). After adjustments, the other included variables (eg, body mass index and cigarette smoking) did not have a significant influence on the risk of endometrial cancer.

Women with a family history of endometrial cancer (mother or sister) had an odds ratio of endometrial cancer of 2.1 ( $95 \%$ confidence interval, 1.1-3.8). 
Table IV. Risk factors for endometrial cancer in women $\measuredangle 50$ years old

\begin{tabular}{|c|c|c|c|c|c|}
\hline Risk factor & $\begin{array}{l}\text { Case patients } \\
(n=237)\end{array}$ & $\begin{array}{l}\text { Control subjects } \\
\quad(n=538)\end{array}$ & $\begin{array}{l}\text { Crude } \\
\text { odds ratio }\end{array}$ & $\begin{array}{l}\text { Adjusted } \\
\text { odds ratio* }\end{array}$ & $\begin{array}{l}95 \% \text { Confidence } \\
\text { interval }\end{array}$ \\
\hline \multicolumn{6}{|c|}{ Family disposition } \\
\hline No & 194 & 471 & $1 \dagger$ & $1 \dagger$ & - \\
\hline Yes & 23 & 31 & 1.8 & 2.1 & $1.1-3.8$ \\
\hline Unknown & 20 & 36 & 1.4 & 2.1 & $1.2-3.8$ \\
\hline \multicolumn{6}{|l|}{ Parity } \\
\hline 0 & 71 & 91 & $1 \dagger$ & $1 \dagger$ & - \\
\hline 1 & 32 & 88 & 0.5 & 0.6 & $0.3-1.1$ \\
\hline 2 & 86 & 243 & 0.5 & 0.3 & $0.2-0.6$ \\
\hline$\geq 3$ & 48 & 116 & 0.5 & 0.2 & $0.1-0.4$ \\
\hline \multicolumn{6}{|l|}{ Age at first birth } \\
\hline$\leq 22 y$ & 79 & 55 & $1 \dagger$ & $1 \dagger$ & - \\
\hline $23-25 y$ & 96 & 100 & 0.7 & 0.5 & $0.3-0.8$ \\
\hline $26-29 y$ & 30 & 178 & 0.1 & 0.1 & $0.04-0.1$ \\
\hline$\geq 30 y$ & 5 & 150 & 0.02 & 0.02 & $0.01-0.05$ \\
\hline \multicolumn{6}{|c|}{ Abortions, induced } \\
\hline 0 & 205 & 395 & $1 \dagger$ & $1 \dagger$ & - \\
\hline 1 & 24 & 105 & 0.4 & 0.5 & $0.3-0.9$ \\
\hline$\geq 2$ & 8 & 38 & 0.4 & 0.4 & $0.2-0.8$ \\
\hline \multicolumn{6}{|c|}{ Duration of oral contraceptives } \\
\hline Never used & 90 & 751 & $1 \dagger$ & $1 \dagger$ & - \\
\hline$<1$ y & 52 & 95 & 0.5 & 0.4 & $0.3-0.7$ \\
\hline $1-5 y$ & 50 & 210 & 0.2 & 0.2 & $0.1-0.3$ \\
\hline$>5 y$ & 45 & 158 & 0.2 & 0.2 & $0.1-0.4$ \\
\hline \multicolumn{6}{|c|}{$\begin{array}{l}\text { Duration of hormone replacement } \\
\text { therapy }\end{array}$} \\
\hline Never used & 198 & 488 & $1 \dagger$ & $1 \dagger$ & - \\
\hline$<1$ y & 17 & 33 & 1.3 & 1.6 & $0.8-3.0$ \\
\hline $1-5 y$ & 17 & 13 & 3.2 & 3.1 & $1.4-7.0$ \\
\hline$>5 y$ & 5 & 4 & 3.1 & 2.8 & $0.7-11.6$ \\
\hline
\end{tabular}

*Variables in multivariate analysis included age, family history of endometrial cancer, body mass index, diabetes mellitus, hypertension, menarche, pregnancy, number of pregnancies, number of births, number of induced abortions, age at first birth, hyperandrogenism, amenorrhea, oral contraceptive use, hormone replacement therapy, cigarette smoking, and years of schooling.

†Reference category.

With respect to reproductive history, after completion of 1 term pregnancy the risk of development of endometrial cancer was reduced by $40 \%$ of the risk of nulliparous women (odds ratio, 0.6; $95 \%$ confidence interval, 0.3 1.1). The risk of endometrial cancer decreased significantly with increasing age at first birth (Table IV). Induced abortions also decreased the risk of endometrial cancer (after $\geq 2$ abortions odds ratio, $0.4 ; 95 \%$ confidence interval, $0.2-0.8$ ).

U se of oral contraceptives for 1 to 5 years decreased the risk of endometrial cancer (odds ratio, $0.2 ; 95 \%$ confidence interval, 0.1-0.3) compared with the risk in women who had never received oral contraceptives. Use of oral contraceptives for $>5$ years did not further decrease the risk.

More case patients (16.5\%) than control subjects (9.3\%) had received hormone replacement therapy. After confounders were controlled for, the odds ratio of endometrial cancer among women who received hormone replacement therapy for 1 to 5 years was increased 3.1 times (95\% confidence interval, 1.4-7.0). Hormone replacement therapy for $>5$ years did not increase the risk of endometrial cancer further.

Because the case patients included in this study were all associated with incident events in Denmark during the period 1987 through 1994, absolute incidence rates could be calculated (Fig 1). An exponential increase in risk with increasing age was demonstrated.

\section{Comment}

Generally, the etiology of endometrial cancer is multifactorial. The pathogenesis may be explained by the estrogen theory. Increased availability of estrogen to the estrogen sensitive endometrium increases the risk of endometrial cancer. Thus the contribution of different risk factors is explained by their separate and mutual effects on the plasma estrogen concentration.

The main source of plasma estrogen in premenopausal women is ovarian production. In postmenopausal women the primary source of estrogen is the extraglandular conversion of androstenedione to estrone and estradiol. This aromatization occurs in adipose tissue, which is particularly rich in enzymes facilitating this process. In postmenopausal women the concentration of plasma estrogen is reduced by $70 \%$ to $80 \%$ with respect to the premenopausal level.

Overweight status. In this analysis, after we controlled for confounding influences from all other risk factors, 
body mass index did not show an independent effect in development of endometrial cancer. The other risk factors controlled for included family predisposition. To the extent that overweight status is related to family predisposition, this risk was eliminated.

Numerous studies have concurrently shown overweight status to be a risk factor for endometrial cancer among postmenopausal women, ${ }^{1-4}$ and only a few studies have not confirmed this association. 15 Most studies on premenopausal women have demonstrated overweight status and, in particular, obesity to be risk factors for endometrial cancer. $8,9,11$ Levi et al ${ }^{3}$ investigated body mass at different ages and subsequent endometrial cancer risk and found the risk estimates to be substantially higher at older ages. A possible reason that body mass index showed a weaker effect in young women is that the ovarian estrogen production in premenopausal women highly exceeds the production in adipose tissue and consequently dominates the estrogenic influence.

Hormone replacement therapy. The increased risk of endometrial cancer among women who received hormone replacement therapy is in accordance with other previously conducted studies.15, 16 In Denmark most young women who receive hormone replacement therapy are prescribed an estrogen-plus-progestogen regimen, whereas $<\%$ use estrogen-only products. Although we had no information about the specific types of hormone replacement therapy used by the case patients and control subjects, this study suggests that combination regimens do not ensure an unchanged risk of endometrial cancer with respect to women who have never taken hormones.

We found 4 studies that assessed the influence of hormone replacement therapy among women $<50$ years old. ${ }^{7-9,} 11$ In all of these studies the therapy was described as estrogen replacement therapy. None of the studies discriminated between different hormone regimens.

Oral contraceptives. The significantly decreased risk of endometrial cancer among women who had used oral contraceptives for 1 to 5 years again demonstrates that oral contraceptives not only protect against endometrial cancer among current users but also decrease the risk years after cessation of oral contraceptive use. We expected our odds ratios to be lower than those reported in studies assessing the influence of oral contraceptive use on the risk of endometrial cancer among women in the postmenopausal period because the protection does fade with time.

Our results concerning the influence on premenopausal endometrial cancer are in accordance with previously conducted studies on this effect. Henderson et al ${ }^{9}$ found that use of combination oral contraceptives for 4 to 6 years implied an odds ratio of premenopausal endometrial cancer of 0.14 .

The protective influence among current users of oral

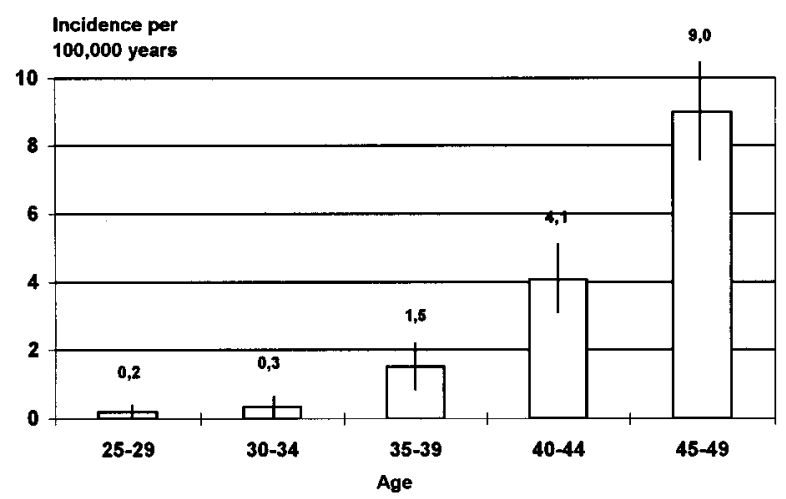

Fig 1. Incidence of endometrial cancer among young women in Denmark. Error bars, 95\% confidence intervals.

contraceptives is probably because of the relatively high progestogen level in these preparations. The decreased risk many years after cessation has not been explained.

Reproduction. After adjustment for all other variables, a clearly decreasing risk with increasing number of births was demonstrated. Incomplete pregnancies, such as induced and spontaneous abortions, were also associated with a significant protection. The most striking protective effect was demonstrated by late age at first birth.

These results are consistent with the results of other studies of both premenopausal and postmenopausal women. ${ }^{7,} 9$ The influence from reproductive parameters is more evident in the younger age groups. ${ }^{7}$ The protective effect of parity is in agreement with the unopposed estrogen theory; pregnancy is a time during which the progestogen/ estrogen ratio is relatively high.

In the analysis the estimates according to age at first birth were adjusted for age at diagnosis. Therefore the increasing protection with increasing age at first birth cannot be explained by the increasing incidence rate of endometrial cancer by age. Possibly the more effective progestogen production among young women may explain why pregnancy-related endogenous hormones have less influence than in older women of fertile age, among whom the ovarian progestogen production is less effective.

Menarche and amenorrhea. We found no relationship between early menarche and endometrial cancer after adjustment for other confounders, including overweight status. Previous studies on premenopausal women have demonstrated either higher risk estimates if menarche occurred early ${ }^{8}$ or no significant relationship at all. ${ }^{9}$

The crude association between periods of amenorrhea and risk of endometrial cancer disappeared after we controlled for the other included confounders. The increased risk associated with amenorrhea could be a result of the association between amenorrhea and infertility or nulliparity. When the latter variables were controlled for, no effect of amenorrhea per se could be demonstrated. 
Table V. Etiologic fraction of endometrial cancer in young women

\begin{tabular}{lccr}
\hline \multicolumn{1}{c}{ Risk factor } & Odds ratio & Prevalence & Etiologic fraction (\%) \\
\hline Family predisposition to endometrial cancer & 2.1 & 0.06 & 6.1 \\
Parity $>1$ & 0.3 & 0.67 & -88.3 \\
Age at first birth $>30$ y & 0.02 & 0.28 & -37.8 \\
Induced abortions $\geq 1$ & 0.5 & 0.27 & -15.6 \\
Duration of oral contraceptive use 1-5 y & 0.2 & 0.39 & -45.4 \\
Duration of hormone replacement therapy 1-5 y & 3.1 & 0.02 & 4.0 \\
\hline
\end{tabular}

Prevalence represents prevalence of respective risk factor among women in control group. Etiologic fraction indicates negative impact or protective impact of different exposures ( risk factors) expressed in terms of how much each exposure increases or reduces occurrence of endometrial cancer compared with a situation without the existence of that particular exposure.

Some studies have demonstrated amenorrhea to be a risk factor for endometrial cancer among premenopausal women, 8,9 whereas other studies have not found any significant association between oligomenorrhea or amenorrhea $^{2}$ and endometrial cancer.

Family predisposition. Family history of endometrial cancer in a first-degree relative (mother or sister) is a well-documented risk factor for endometrial cancer among both premenopausal and postmenopausal women, with odds ratios between 1.5 and 2.8.17, 18 This relationship was confirmed by our data. It should be noted that the estimated odds ratio of 2.1 represents the impact of family predisposition after body mass index is controlled for. Thus the increased risk was caused by genetic circumstances other than adiposity.

Diabetes and hypertension. In this study no significant association emerged between endometrial cancer and diabetes; however, it should be noted that only 4 case patients $(1.7 \%)$ reported diabetes. Some studies have found diabetes to be a risk factor for endometrial cancer among premenopausal women, 19 whereas other studies have not been able to confirm this association. ${ }^{20-22}$ The influence has been explained by higher levels of estrone and lipids in the plasma of women with diabetes. 9

$\mathrm{H}$ ypertension was not an independent risk factor. Some studies have reported an odds ratio as great as 2.1 among postmenopausal women, possibly as a result of an association between hypertension and body weight. ${ }^{1}$ 0 ther studies have not been able to demonstrate this association. ${ }^{2}$

Cigarette smoking. In this study we were not able to demonstrate an independent effect of smoking. Numerous studies on the cancerogenic effect of cigarette smoking on female reproductive organs have been done. There has been particular focus on the relationship between endometrial cancer and smoking because of the well-documented antiestrogenic effect of smoking. The tobacco metabolites facilitate the production of biologically less active estrogens. Clinically, this is concordant with the observation that women who are current smokers have a higher incidence of osteoporosis and enter menopause at an earlier age. ${ }^{23}$

All studies have demonstrated a protective effect of cig- arette smoking on the risk of development of endometrial cancer among postmenopausal women. Odds ratios of 0.4 to 0.8 have been reported for current smokers, with an inverse dose-response relationship to the number of cigarettes per day. ${ }^{24}$ The protection decreases gradually with time after cessation of smoking. ${ }^{24}$ It is remarkable that the protective influence of cigarette smoking has not been apparent in several studies on premenopausal women with endometrial cancer. ${ }^{11,24}$

Education. After adjustment for all variables, we could not retrieve an association with years of schooling, which is a reliable indicator of social class. Several studies have demonstrated an increased risk among postmenopausal women in high socioeconomic classes ${ }^{7}$ after adjustments for supposed increased consumption of estrogen replacement therapy and lower parity among highly educated women.

Etiologic fraction. To put the estimated odds ratios in perspective the prophylactic potential was calculated for each risk factor; this is the so-called etiologic fraction, which indicates how much each protective risk factor reduces the occurrence of endometrial cancer and how much each risk factor increases the occurrence with respect to a situation in which there had been no exposure to these risk factors (Table $\mathrm{V}$ ). The calculations were based on the adjusted odds ratio and the prevalence of each risk factor among women in the control population.

With a mother or sister who had endometrial cancer, the risk of development of endometrial cancer was increased by a factor of 2 . The prevalence was around $6 \%$, and the corresponding etiologic fraction was $6 \%$. $\mathrm{H}$ ormone replacement therapy increased risk by a factor of 3 , but only about $2 \%$ of premenopausal women receive hormone replacement therapy. Consequently, the etiologic fraction of hormone replacement therapy was $4 \%$. Oral contraceptive use for $>1$ year, $\geq 2$ term pregnancies, age $\geq 30$ years when giving birth at first time, and a history of incomplete pregnancies are all conditions that decreased the risk of development of endometrial cancer. Their respective etiologic fractions were $-45 \%$, $-88 \%,-38 \%$, and $-16 \%$, suggesting that these circumstances significantly reduce the occurrence of endometrial cancer among young women. 
In conclusion, a number of risk factors for endometrial cancer are common to premenopausal and postmenopausal women. These are family history of endometrial cancer, reproductive history, hormone replacement therapy, and the use of oral contraceptives.

When we considered the influence of the different risk factors among young women in this study, the reproductive variables were dominant. Increasing number of births reduced the risk of endometrial cancer. If all women gave birth $\geq 2$ times, about $40 \%$ of endometrial cancer in the premenopausal age group could be eliminated.

As a consequence of the well-documented protective effects of oral contraceptive use against endometrial cancer and when the high percentage of women who have ever taken oral contraceptives is taken into account, one would expect a significantly decreasing trend in endometrial cancer among young women during the last 15 years. However, such a decrease has not occurred in Denmark. ${ }^{1}$ The explanation could be a counterbalancing decreasing number of births and possibly a change in dietary composition with an increased sugar intake. 25

Noncontraceptive hormones are seldom used by young women. Even though these hormones tripled the risk when used for $>1$ year, the impact of hormone replacement therapy on endometrial cancer among premenopausal women remained small.

\section{REFERENCES}

1. Ewertz M, Schou G, Boice JD Jr. The joint effect of risk factors on endometrial cancer. Eur J Cancer Clin Oncol 1988;24:18994.

2. Brinton $L A$, Berman $M L$, Mortel $R$, Twiggs $L B, B$ arrett RJ, Wilbanks GD, et al. Reproductive, menstrual, and medical risk factors for endometrial cancer: results from a case-control study. Am J Obstet Gynecol 1992;167:1317-25.

3. Levi F, Lavecchia C, Negri E, Parazzini F, Francecchi S. Body mass at different ages and subsequent endometrial cancer risk. Int J Cancer 1992;50:567-71.

4. Jick SS, Walker AM, Jick H. O ral contraceptives and endometrial cancer. Obstet Gynecol 1993;82:931-5.

5. Brinton LA, H oover RN. Estrogen replacement therapy and endometrial cancer risk: unresolved issues. The Endometrial Cancer Collaborative Group. O bstet Gynecol 1993;81:265-71.

6. Combination oral contraceptive use and the risk of endometrial cancer. The Cancer and Steroid H ormone Study of the Centers for Disease Control and the National Institute of Child Health and Human Development. JAMA 1987;257:796-800.
7. Parazzini F, La Vecchia C, Negri E, Fedele L, Balotta F. Reproductive factors and risk of endometrial cancer. Am J O bstet Gynecol 1991;164:522-7.

8. La Vecchia C, Franceschi S, Decarli A, Gallus G, Tognoni G. Risk factors for endometrial cancer at different ages. J Natl Cancer Inst 1984;73:667-71.

9. Henderson BE, Casagrande JT, Pike MC, Mack T, Rosario I, Duke A. The epidemiology of endometrial cancer in young women. Br J Cancer 1983;47:749-56.

10. Smith EM, Sowers MF, Bums TL. Effects of smoking on the development of female reproductive cancers. J Natl Cancer Inst 1984;73:371-6.

11. Tyler CW Jr, Webster LA, Ory HW, Rubin G. Endometrial cancer: how does cigarette smoking influence the risk of women under age 55 years having this tumor? Am J O bstet Gynecol 1985;151:899-905.

12. Endometrial cancer and combined oral contraceptives. The WHO Collaborative Study of Neoplasia and Steroid Contraceptives. Int J Epidemiol 1988;17:263-9.

13. Dahlgren $E$, Johansson $S$, O dén $A$, Lindström $B$, Janson PO. A model for prediction of endometrial cancer. Acta Obstet Gynecol Scand 1989;68:507-10.

14. Klein JP, Keiding N, Kreiner S. Graphical models for panel studies, illustrated on data from the Framingham H eart Study. Stat Med 1995;14:1265-90.

15. Koumantaki Y, Tzonou A, Koumantakis E, Kaklamani E, Aravantinos D, Trichopoulos D. A case-control study of cancer of endometrium in Athens. Int J Cancer 1989;43:795-9.

16. Paganini-Hill A, Ross RK, Henderson BE. Endometrial cancer and patterns of use of estrogen replacement therapy: a cohort study. Br J Cancer 1989;59:445-7.

17. Gruber SB, Thompson WD. A population-based study of endometrial cancer and familial risk in younger women. Cancer and Steroid Hormone Study Group. Cancer Epidemiol Biomarkers Prev 1996;5:411-7.

18. Parazzini F, La Vecchia C, Moroni S, Chatenoud L, Ricci E. Family history and the risk of endometrial cancer. Int J Cancer 1994;59:460-2.

19. Geisler $\mathrm{HE}$, Huber $\mathrm{CP}$, Roger $\mathrm{S}$. Carcinoma of the endometrium in premenopausal women. Am J Obstet Gynecol 1969;104:65763.

20. Gitsch G, Hanzal E, Jensen D, Hacker NF. Endometrial cancer in premenopausal women 45 years and younger. O bstet Gynecol 1995;85:504-8.

21. Gallup DG, Stock RJ. Adenocarcinoma of the endometrium in women 40 years of age or younger. O bstet Gynecol 1984;64:41720.

22. Kaku T, Matsuo K, Tsukamoto N. Endometrial carcinoma in women aged 40 years or younger: a Japanese experience. Int J Gynecol Cancer 1993;3:147-53.

23. Baron JA, La Vecchia C, Levi F. The antiestrogenic effect of cigarette smoking in women. Am J Obstet Gynecol 1990;162:50214.

24. Weir HK, Sloan M, Kreiger N. The relationship between cigarette smoking and the risk of endometrial neoplasms. Int J Epidemiol 1994;23:261-6.

25. Levi F, Franceschi S, Negri E, La Vecchia C. Dietary factors and the risk of endometrial cancer. Cancer 1993;71:3575-81. 